\title{
IMPLEMENTATION WITH INCOMPLETE INFORMATION IN EXCHANGE ECONOMIES
}

\author{
By Thomas R. Palfrey and SANJay SRIVASTaVA ${ }^{1}$
}

\begin{abstract}
In this paper, we analyze the problem of designing incentive compatible mechanisms in pure exchange economic environments when agents have incomplete information. The equilibrium concept employed is Bayesian Nash equilibrium and the notion of implementation is full implementation, which is stronger than the more commonly employed notion of truthful implementation.

An allocation rule is truthfully implementable if there exists a direct mechanism to which truth telling is an equilibrium and which yields the allocation rule as its truthful equilibrium outcome. An allocation rule is fully implementable if there exists a mechanism which yields the allocation rule as its unique equilibrium outcome. More generally, a set of allocation rules, or a social choice set, is fully implementable if there exists a mechanism whose equilibrium outcomes coincide with the set. This stronger notion of implementation avoids the well known problems of multiple equilibria which arise in direct revelation games.

We develop a condition, termed Bayesian monotonicity, which we show is necessary for full implementation. An incentive compatibility condition is also necessary. We prove that Bayesian monotonicity and a slightly stronger incentive compatibility condition are sufficient for full implementation when there are at least three agents.

We present several examples of allocation rules which do and do not satisfy our condition. One example is that of an allocation rule which is fully implementable by an indirect mechanism, but for which every equivalent direct mechanism has multiple equilibrium outcomes.
\end{abstract}

KEYWORDS: Implementation, incomplete information, noncooperative games, incentive compatibility, general equilibrium.

\section{INTRODUCTION}

MOST RESEARCH ON THE DESIGN of incentive compatible mechanisms in economies with incomplete information relies on the revelation principle (see, e.g., Myerson (1979), Harris and Townsend (1981)). The principle states that if an allocation rule is a Bayesian equilibrium outcome to some mechanism, then there is an equivalent direct mechanism to which truth telling is an equilibrium and whose truthful equilibrium yields the same outcome. In this case, the direct mechanism is said to truthfully implement the allocation rule (Dasgupta, Hammond, and Maskin (1979)). The revelation principle has frequently been invoked to argue that in designing incentive compatible mechanisms, no loss of generality is incurred by restricting attention to direct mechanisms.

In this paper, we study the problem of designing incentive compatible mechanisms using a notion of implementation stronger than truthful implementation. In particular, an allocation rule is said to be fully implementable (Dasgupta, Hammond, and Maskin (1979)) if there exists a mechanism which yields the

\footnotetext{
${ }^{1}$ We thank Andrew Postlewaite, participants of the NBER/CEME/NSF Conference on General Equilibrium Theory, Cambridge, May 1985, and the Northwestern University 1985 Summer Workshop on Strategic Behavior and Competition, for their feedback on earlier versions of this work. We also thank David Kreps and an anonymous referee for helpful suggestions which have greatly improved the paper.
} 
allocation rule as its unique equilibrium outcome. More generally, a set of allocation rules, or a social choice set (SCS), is iully implementable if there exists a mechanism whose equilibrium outcomes coincide with the SCS.

The primary reason for examining the implications of this stronger notion of implementation is that it avoids well-known problems associated with multiple equilibria. For instance, it is possible to construct examples in which an indirect mechanism has a unique equilibrium outcome but for which every equivalent direct mechanism has more than one equilibrium outcome (see Postlewaite and Schmeidler (1986) and Dasgupta, Hammond, and Maskin (1979)). A second type of problem arises when an indirect mechanism has multiple equilibrium outcomes; application of the revelation principle requires the construction of a different direct mechanism for each outcome of the original mechanism. Third, many direct mechanisms studied in the literature have multiple equilibrium outcomes (e.g. Milgrom (1981), Demski and Sappington (1984), and Rob (1984)), and the question arises whether indirect mechanisms can eliminate some of these equilibria.

The problem of full implementation has been studied extensively when agents have complete information. Maskin (1977) identified a condition on SCS's called monotonicity, which is necessary for full implementation in Nash equilibrium, and, in economic environments, is sufficient if there are at least three agents. This paper provides a general characterization of (Bayesian Nash) fully implementable allocation rules in pure exchange economic settings where individual agents have incomplete and asymmetric information. We show that a condition, termed Bayesian monotonicity, is necessary for implementation as is an incentive compatibility condition. We also show that when there are at least three agents, this condition and a slightly stronger incentive compatibility condition are sufficient to fully implement an allocation rule. The Bayesian monotonicity condition reduces to Maskin's familiar monotonicity condition when there is complete information.

Postlewaite and Schmeidler (1986) have studied this problem for the special case when an informational restriction, called nonexclusivity of information, is imposed. This restriction requires that with $I$ agents, every group of $I-1$ agents collectively has complete information. They establish that a version of monotonicity is necessary for implementation, and that a second, stronger monotonicity condition is sufficient with three or more agents. While this result is an important first step, their informational restriction excludes many models of interest, such as those commonly employed to study auctions (e.g. Myerson (1981)), public goods provision (e.g. Laffont and Maskin (1982)), models of optimal trading mechanisms with incomplete information (e.g. Myerson and Satterthwaite (1983)), delegation games (e.g. Kalai and Samet (1986)), and partnership arrangements (e.g. Cramton, Gibbons, and Klemperer (1987)). In these models and in most others, agents have exclusively private information.

Our characterization goes beyond that of Postlewaite and Schmeidler (1986) in two important ways. First, we allow for very general information structures, ranging from exclusively private information to complete information. As such, it 
contains as special cases previously known results about Nash and Bayesian Nash implementation in economic environments, and, in addition, our results cover the important set of problems listed above. Second, we do not impose a stronger version of monotonicity in proving sufficiency, so our results are not only broader in scope but provide a tighter characterization.

\section{THE MODEL AND DEFINITION}

The set of economic states (or environments) is denoted by a finite set, $S$, $S=\{1,2, \ldots, S\}$. Each $s \in S$ describes the set of agents, their endowments, and their preferences. Following Postlewaite and Schmeidler (1986), we assume that the number of agents and the aggregate endowment are independent of the state. This assumption implies that the feasible set is independent of the state and is in keeping with most studies of implementation in Nash equilibrium in games of complete information. We denote by $I$ the number of agents and by $\bar{w}$ the aggregate endowment in any state, $\bar{w} \in \mathscr{R}^{L}, \bar{w}>0$, where $\mathscr{R}$ is the real line. Note that these assumptions allow individual endowments to vary across states.

$U^{i}: \mathscr{R}_{+}^{L} \times S \rightarrow \mathscr{R}$ is the utility function of agent $i$ in state $s$, and is assumed to be strictly increasing and bounded below for each $s$. Let

$$
\begin{aligned}
& A=\left\{z \in \mathscr{R}_{+}^{L I} \mid \sum_{i=1}^{I} z^{i} \leq \bar{w}\right\}, \quad \text { and let } \\
& X=\{x: S \rightarrow A\}
\end{aligned}
$$

be the set of all feasible allocation rules. Note that $0 \in A$, so that it is feasible to destroy the social endowment. A Social Choice Set (SCS), denoted $F$, is a subset of $X$.

An agent's information is represented by a partition $\Pi^{i}$ of $S$. Elements of $I I^{i}$ are called events, and each event $E^{i} \in \Pi^{i}$ is a maximal set of states that agent $i$ cannot distinguish. In particular, if the state is $s$, we assume that agent $i$ knows only that the true state lies in a set $E^{i}(s) \subset S$. Each agent also has a prior distribution over states, denoted by $q^{i}$, and we assume that $q^{i}(s)>0$ for all $i, s$. Complete information is the special case $E^{i}(s)=\{s\}$ for all $i$ and $s$. Let $\Pi=\left\{\Pi^{1}, \Pi^{2}, \ldots, \Pi^{I}\right\}$.

We assume that the partitions, the priors, the set of utility functions, and the aggregate endowment are all common knowledge. Given $x \in X$ and an event $E^{i}(s)$, agent $i$ evaluates the expected utility of $x^{i}$ after updating his prior probability on states using Bayes rule. The posterior probability is given by

$$
q^{i}\left(t \mid E^{i}(s)\right)= \begin{cases}0 & \text { if } t \notin E^{i}(s), \\ \frac{q^{i}(t)}{q^{i}\left(E^{i}(s)\right)} & \text { if } t \in E^{i}(s) .\end{cases}
$$


The interim expected utility of allocation $x$ to agent $i$ at state $s$ is

$$
\sum_{t \in E^{i}(s)} q^{i}\left(t \mid E^{i}(s)\right) U^{i}\left(x^{i}(t), t\right)
$$

We can now define an interim preference relation on $X, R^{i}$, by

$$
\begin{aligned}
& x R^{i}\left(E^{i}(s)\right) y \quad \text { if and only if } \\
& \sum_{t \in E^{i}(s)} q^{i}\left(t \mid E^{i}(s)\right) U^{i}\left(x^{i}(t), t\right) \geq \sum_{t \in E^{i}(s)} q^{i}\left(t \mid E^{i}(s)\right) U^{i}\left(y^{i}(t), t\right) .
\end{aligned}
$$

Let $P^{i}\left(E^{i}(s)\right)$ denote the strict preference relation derived from $R^{i}\left(E^{i}(s)\right)$.

$$
\text { Assumption 1: For all } s \in S, \bigcap_{i} E^{i}(s)=\{s\} \text {. }
$$

This assumption, made with essentially no loss of generality, states that pooled information is complete, i.e. there are no redundant states. An implication of this assumption is that for any $E^{1}, \ldots, E^{I}$, if $E^{i} \in \Pi^{i}$ for all $i$ and $\bigcap_{i} E^{i} \neq \varnothing$ then $\bigcap_{i} E^{i}$ is a singleton. To see this, suppose $s_{1}, s_{2} \in \cap E^{i}, s_{1} \neq s_{2}$. Since $E^{i} \in \Pi^{i}$ and each $s \in S$ lies in exactly one event in $\Pi^{i}$, this implies $s_{2} \in E^{i}\left(s_{1}\right)$ for all $i$, so that $s_{1}, s_{2} \in E^{i}\left(s_{1}\right)$ for all $i$, contradicting Assumption 1. We also limit attention to SCS's which are closed under the common knowledge concatenation defined by $\Pi$; this also entails no loss of generality (see Postlewaite and Schmeidler (1986)) and is defined as follows. Denote by $\Pi^{*}$ the common knowledge partition defined by $\Pi$.

Assumption 2: For any $x_{1}, x_{2} \in F$, for any $E \in \Pi^{*}$, if

$$
y(t)= \begin{cases}x_{1}(t) & \text { if } t \in E, \\ x_{2}(t) & \text { if } t \notin E,\end{cases}
$$

then $y \in F$.

Here, $y$ is a common knowledge concatenation of $x_{1}$ and $x_{2}$, and the assumption says that $F$ is closed under such concatenations.

This completes our description of the basic model, and we turn next to a description of mechanisms or the rules according to which trade takes place after agents receive their private information. A mechanism consists of a message space for each agent and an outcome function which maps the aggregate message space to the set of outcomes. A strategy for an agent is a decision rule which maps his private information into his message space. The concept of equilibrium is Bayesian Nash equilibrium. Formally:

Definition 1: A mechanism is a pair $(M, g), \quad M=M^{1} \times M^{2} \ldots \times M^{I}$, $g: M \rightarrow A$. If $M^{i}=\Pi^{i}$ for all $i$, then $(M, g)$ is a direct mechanism. 
Definition 2: A strategy for agent $i$ is $\sigma^{i}: \Pi^{i} \rightarrow M^{i}$.

For any state $s$, we use the following notation:

$$
\begin{aligned}
& \sigma(E(s))=\left\{\sigma^{1}\left(E^{1}(s)\right), \ldots, \sigma^{I}\left(E^{I}(s)\right)\right\} ; \sigma=\left\{\sigma^{1}, \ldots, \sigma^{I}\right\} ; \\
& \sigma^{-i}=\left\{\sigma^{1}, \ldots, \sigma^{i-1}, \sigma^{i+1}, \ldots, \sigma^{I}\right\} ; \text { and } \\
& g(\sigma)=\{g(\sigma(E(1))), \ldots, g(\sigma(E(S)))\} .
\end{aligned}
$$

Definition 3: $\sigma$ is a (Bayesian Nash) equilibrium to $(M, g)$ if, for all $i$ and $s, g(\sigma) R^{i}\left(E^{i}(s)\right) g\left(\sigma^{-i}, \tilde{\sigma}^{i}\right)$ for all $\tilde{\sigma}^{i}: \Pi^{i} \rightarrow M^{i}$.

Definition 4: A mechanism $(M, g)$ fully implements $F$ if (i) for any $x \in F$, there exists an equilibrium, $\sigma$, with $g(\sigma)=x$; (ii) if $\sigma$ is an equilibrium, then $g(\sigma) \in F$.

Part (i) requires that every allocation rule in $F$ is an equilibrium outcome of the mechanism, while (ii) requires that all equilibrium outcomes lie in $F$.

If there exists a mechanism $(M, g)$ which fully implements $F$, then $F$ is said to be implementable. In the next Section, we present a condition on SCS's, called Bayesian monotonicity, which is an extension of Maskin's (1977) notion of monotonicity. We also show that Bayesian monotonicity and an incentive compatibility condition are necessary for implementation. In Section 4, we show that with at least three agents, Bayesian monotonicity and a slightly stronger incentive compatibility condition are sufficient for implementation.

\section{NECESSARY CONDITIONS FOR IMPLEMENTATION:} MONOTONICITY AND SELF-SELECTION

\section{A. Preliminary Definitions}

If an SCS is fully implemented by a mechanism, then all the equilibrium outcomes to that mechanism are elements of the SCS. We thus need to develop notation to keep track of all possible equilibrium strategies. For our purposes, a special type of strategy, called a deceptive strategy, turns out to be crucial to the analysis. To motivate what we mean by deceptive strategies, we begin by considering a direct mechanism, so $M^{i}=\Pi^{i}$ for all $i$. Truth telling is simply the strategy $\sigma^{i}\left(E^{i}(s)\right)=E^{i}(s)$ for all $i$ and $s$. If all agents act truthfully, the intersection of their reported events at $s$ is $\bigcap_{i} E^{i}(s)$, which, by Assumption 1, is simply $\{s\}$. Next, we give the following definition.

Definition 5: A deception for $i$ is a function $\alpha^{i}: \Pi^{i} \rightarrow \Pi^{i}$.

The set of all deceptions for $i$ is equivalent to the set of all possible strategies available to $i$ in a direct mechanism, where the truth-telling strategy is simply the identity deception. Suppose in a direct mechanism each $i$ is using deception $\alpha^{i}$, so 
that at $s$, each $i$ reports $\alpha^{i}\left(E^{i}(s)\right)$ instead of $E^{i}(s)$. For each $s$, two possibilities arise: (a) $\bigcap_{i} \alpha^{i}\left(E^{i}(s)\right) \neq \varnothing$ and (b) $\bigcap_{i} \alpha^{i}\left(E^{i}(s)\right)=\varnothing$. In case (a), the discussion following Assumption 1 yields that the intersection is a singleton. Further, in this case, it is impossible to detect any inconsistency in the reports despite the deceptions. In case (b), the reports are clearly incompatible (though it may be impossible to infer which agent or group of agents is "causing" the incompatibility). In general, some group deceptions will lead to compatible reports and some will not. A major component of the implementation problem is to identify which group deceptions are consistent with equilibrium behavior. The problem is simplified by the fact that it is fairly easy to construct mechanisms to ensure that no equilibrium will involve incompatible deceptions. Formally, we have the following definition.

Definition 6: A group deception $\alpha=\left\{\alpha^{1}, \ldots, \alpha^{I}\right\}$ is compatible with $\Pi$ if for all $s, \bigcap_{i} \alpha^{i}\left(E^{i}(s)\right) \neq \varnothing$.

Assumption 1 yields $\bigcap_{i} \alpha^{i}\left(E^{i}(s)\right) \neq \varnothing$ if and only if it is a singleton, as discussed previously. Accordingly, for any $\alpha$ compatible with $\Pi$, we define

$$
\{\alpha(s)\}=\bigcap_{i} \alpha^{i}\left(E^{i}(s)\right) .
$$

If state $s$ occurs and all agents are using a compatible group deception $\alpha$, then the intersection of their reported events is $\alpha(s)$, and we say that the deceptive strategies agree on $\alpha(s)$ at $s$.

Lemma 1: For any $\alpha$ compatible with $\Pi, t \in E^{i}(s) \Rightarrow \alpha(t) \in E^{i}(\alpha(s))$ for all $i$ and $s$.

Proof: $s^{\prime}=\alpha(s) \Rightarrow \alpha^{i}\left(E^{i}(s)\right)=E^{i}\left(s^{\prime}\right)$ for all $i$. For any $i, t \in E^{i}(s) \Rightarrow E^{i}(t)$ $=E^{i}(s)$, so $\alpha^{i}\left(E^{i}(t)\right)=E^{i}\left(s^{\prime}\right)$, which implies $\alpha(t) \in E^{i}\left(s^{\prime}\right)$.

Q.E.D.

Consider next the effect of a compatible group deception on allocations. Suppose a planner wishes to implement an allocation rule $x$, and designs a direct mechanism whose truthful equilibrium outcome is $x$. Then, at $s$, if each $i$ reports $E^{i}(s)$, the intersection of the reported events is $\bigcap_{i} E^{i}(s)=\{s\}$, and the planner allocates $x(s)$. Now suppose the agents are using a compatible group deception, $\alpha$. Then, at $s$, the intersection of the reported events is $\alpha(s)$, and the planner inadvertently allocates $x(\alpha(s))$ at $s$. Denote

$$
x_{\alpha}=\left(x_{\alpha}(1), \ldots, x_{\alpha}(s)\right) \text { where } x_{\alpha}(t)=x(\alpha(t)) \text { for all } t .
$$

Then, the allocation rule generated by the group deception is $x_{\alpha}$ instead of $x$.

More generally, consider an arbitrary mechanism $(M, g)$ and let $\sigma$ be an equilibrium with $g(\sigma)=x$. Corresponding to a deception $\alpha^{i}$ for $i$, we can define a deceptive strategy for $i$ relative to $\sigma^{i}$ by $\sigma_{\alpha}^{i}\left(E^{i}(s)\right)=\sigma^{i}\left(\alpha^{i}\left(E^{i}(s)\right)\right)$. If each $i$ uses 
$\alpha^{i}$, and $\alpha$ is compatible with $\Pi$, we get $g\left(\sigma_{\alpha}\right)=x_{\alpha}$. The reason this is important for implementation is that if, regardless of the mechanism we use, $\sigma_{\alpha}$ is an equilibrium whenever $\sigma$ is, then $x_{\alpha}$ must lie in $F$ whenever $x$ lies in $F$ in order for $F$ to be implementable. This is essentially the content of our Bayesian monotonicity condition, to which we now turn.

\section{B. Bayesian Monotonicity}

To help motivate and explain the intuition behind the concept of Bayesian monotonicity, we begin with complete information, where $E^{i}(s)=\{s\}$ and $q^{i}\left(s \mid E^{i}(s)\right)=1$ for all $i$ and $s$. For this case, Maskin (1977) identified a necessary condition called monotonicity. The following two alternative formulations of this condition are particularly helpful in illustrating how Bayesian monotonicity emerges as a natural extension of Maskin's condition.

For the first representation of monotonicity with complete information, we write a social choice set as a multivalued mapping (correspondence) $F: S \rightrightarrows A$ rather than as a collection of single valued mappings. The greater generality of the latter representation (used everywhere else in this paper) is important for the case of incomplete information, but is superfluous when there is complete information. This follows from the fact that with complete information, the common knowledge partition defined by $\Pi$ consists of singleton events $E^{*}(s)=$ $\{s\}$. Assumption 2 then yields that every selection from $F$ is an element of $F$, so $F$ can be written as a correspondence from $S$ into $A$. Let $R^{i}(s)$ denote $i$ 's ex post preference relation in state $s$.

Definition 7: $\left(s, s^{\prime}, a\right) \in S \times S \times A$ satisfies the monotonicity hypothesis if, for all $i$, for all $b \in A, a R^{i}(s) b \Rightarrow a R^{i}\left(s^{\prime}\right) b$.

In other words, the monotonicity hypothesis holds for outcome $a$ with respect to states $s$ and $s^{\prime}$ if, for every agent, the lower contour set of $a$ at $s$ is contained in that agent's lower contour set of $a$ at $s^{\prime}$. Observe that it is usually not the case that both $\left(s, s^{\prime}, a\right)$ and $\left(s^{\prime}, s, a\right)$ satisfy the monotonicity hypothesis.

Definition 8: $F$ is monotonic if, for all $\left(s, s^{\prime}, a\right)$ such that $a \in F(s)$, if $\left(s, s^{\prime}, a\right)$ satisfies the monotonicity hypothesis then $a \in F\left(s^{\prime}\right)$.

While this is a very useful and simple representation of monotonicity with complete information a slightly different formulation will better serve the purpose of motivating the much more difficult Bayesian monotonicity condition needed with incomplete information. Since $E^{i}(s)=\{s\}$ for all $i$ and $s$, we have $\alpha^{i}: S \rightarrow S$ for each $i$. Consequently, the only types of compatible deceptions which are relevant with complete information are "unanimous" deceptions in 
which for some $s^{\prime}, \alpha^{i}\left(s^{\prime}\right)=\alpha^{j}\left(s^{\prime}\right)=s$ for all $i, j$. Using this alternative representation, we get the following definition:

DEFinition 9: $(\alpha, x)$ satisfies the monotonicity hypothesis if, for all $i$, for all $s^{\prime}$, for all $y \in X, x R^{i}\left(\alpha\left(s^{\prime}\right)\right) y \Rightarrow x_{\alpha} R^{i}\left(s^{\prime}\right) y_{\alpha}$.

To see that the two formulations are equivalent, recall that with complete information, $x R^{i}\left(\alpha\left(s^{\prime}\right)\right) y$ if and only if $U^{i}\left(x^{i}\left(\alpha\left(s^{\prime}\right)\right), \alpha\left(s^{\prime}\right)\right) \geq U^{i}\left(y^{i}\left(\alpha\left(s^{\prime}\right)\right)\right.$, $\left.\alpha\left(s^{\prime}\right)\right)$ while $x_{\alpha} R^{i}\left(s^{\prime}\right) y_{\alpha}$ if and only if $U^{i}\left(x\left(\alpha\left(s^{\prime}\right)\right), s^{\prime}\right) \geq U^{i}\left(y^{i}\left(\alpha\left(s^{\prime}\right)\right), s^{\prime}\right)$. Letting $s=\alpha\left(s^{\prime}\right)$, the equivalence is immediate. Now we can write the following definition:

Definition 10: $F$ is monotonic if, for all $\alpha$ compatible with $\Pi$ and all $x \in F$, if $(\alpha, x)$ satisfies the monotonicity hypothesis (Definition 9) then $x_{\alpha} \in F$.

We can now show how the second formulation of monotonicity extends to the case of incomplete information. One modification is required to deal with a new possibility which could not arise with complete information. With complete information, if $\alpha$ is compatible with $\Pi$, then $\alpha^{i}$ is uniquely determined by $\alpha^{-i}$. This is not true with incomplete information except in the more special case dealt with by Postlewaite and Schmeidler (1986), in which every group of $I-1$ players collectively has complete information. For that special case, Definition 10 applies directly if we simply replace the ex-post preference relation, $R^{i}(s)$, by its interim version, $R^{i}\left(E^{i}(s)\right)$.

For the general case, the monotonicity hypothesis must be modified to account for the possibility that some information is held exclusively by some agent. This we do as follows.

Define $E^{-i}(s)=\bigcap_{j \neq i} E^{j}(s)$. For any $y \in X$ and for any $E^{i} \in \Pi^{i}$, define

$$
y_{E^{i}}(t)= \begin{cases}y\left(E^{-i}(t) \cap E^{i}\right) & \text { if } E^{-i}(t) \cap E^{i} \neq \varnothing, \\ 0 & \text { otherwise. }\end{cases}
$$

In other words, $y_{E^{i}}$ corresponds to $y_{\alpha}$, where $\alpha^{j}$ is the identity for all $j \neq i$ and $\alpha^{i}$ is the "constant deception," $E^{i}$, with the added proviso that $y_{\alpha}(\varnothing)=0$. Given $\alpha$ compatible with $\Pi$, we let $E_{\alpha}^{-i}(s)=\bigcap_{j \neq i} \alpha^{j}\left(E^{j}(s)\right)$ and make the following definition:

Definition 11: $(\alpha, x)$ satisfies the Bayesian monotonicity hypothesis if, for all $i$, for all $s^{\prime}$, for all $y: S \rightarrow A$,

$$
x R^{i}\left(E^{i}(s)\right) y_{E^{i}\left(\alpha\left(s^{\prime}\right)\right)} \text { for all } s \in E_{\alpha}^{-i}\left(s^{\prime}\right) \text { implies } x_{\alpha} R^{i}\left(E^{i}\left(s^{\prime}\right)\right) y_{\alpha} .
$$

Definition 12: $F$ is Bayesian monotonic if, for all $\alpha$ compatible with $\Pi$ and all $x \in F$, if $(\alpha, x)$ satisfies the Bayesian monotonicity hypothesis then $x_{\alpha} \in F$. 
The proof that Bayesian monotonicity is necessary for full implementation in Bayesian equilibrium is now immediate.

\section{ThEOREM 1: If $F$ is implementable then $F$ is Bayesian monotonic.}

Proof: Let $(M, g)$ implement $F$, let $\sigma$ be an equilibrium with $g(\sigma)=x \in F$, and suppose the Bayesian monotonicity hypothesis is satisfied by $(\alpha, x)$ for some $\alpha$ compatible with $\Pi$. We argue that $\sigma_{\alpha}$ defined by $\sigma_{\alpha}^{i}\left(E^{i}(s)\right)=\sigma^{i}\left(\alpha^{i}\left(E^{i}(s)\right)\right.$ for all $s$ is also an equilibrium. Suppose $\sigma_{\alpha}$ is not an equilibrium. Then, there exists $i, s^{\prime}$, and $m^{i} \in M^{i}$ with $g\left(\sigma_{\alpha}^{-i}, m^{i}\right) P^{i}\left(E^{i}\left(s^{\prime}\right)\right) g\left(\sigma_{\alpha}\right)$. Let $\bar{\sigma}^{i}$ be the constant strategy $\bar{\sigma}^{i}\left(E^{i}(t)\right)=m^{i}$ for all $t$. Let $y=g\left(\sigma^{-i}, \bar{\sigma}^{i}\right)$, so $y_{\alpha}=g\left(\sigma_{\alpha}^{-i}, \bar{\sigma}^{i}\right)$ and $y_{\alpha} P^{i}\left(E^{i}\left(s^{\prime}\right)\right) x_{\alpha}$ by construction. The hypothesis yields $s \in E_{\alpha}^{-i}\left(s^{\prime}\right)$ with $y_{E^{i}\left(\alpha\left(s^{\prime}\right)\right)} P^{i}\left(E^{i}(s)\right) x$. Since $\bar{\sigma}^{i}$ is constant, we have $y_{E^{i}\left(\alpha\left(s^{\prime}\right)\right)}(t)=y(t)$ if $E^{-i}(t) \cap$ $E^{i}\left(\alpha\left(s^{\prime}\right)\right) \neq \varnothing$ and $y_{E^{i}\left(\alpha\left(s^{\prime}\right)\right)}(t)=0 \leq y(t)$ otherwise. This implies $y \geq y_{E^{i}\left(\alpha\left(s^{\prime}\right)\right) \text {. }}$ Therefore $y R^{i}\left(E^{i}(s)\right) y_{E^{i}\left(\alpha\left(s^{\prime}\right)\right)}$ so $y P^{i}\left(E^{i}(s)\right) x$. This contradicts $\sigma$ being an equilibrium, and we conclude that $\sigma_{\alpha}$ is an equilibrium, in which case $x_{\alpha}$ is an equilibrium outcome. Since $F$ is implementable, we have $x_{a} \in F$, as required by Bayesian monotonicity.

Q.E.D.

The above proof simplifies somewhat if information is such that every $\alpha$ is compatible. For example, consider the most commonly used class of models in which a state is a vector of types, $s=\left(s^{1}, \ldots, s^{I}\right)$, and each agent knows his own type but receives no precise information about the types of others. If $S^{i}$ denotes the set of types for agent $i$, we get $E^{i}(s)=\left\{s^{i}\right\} \times S^{-i}$. With such diffuse information structures, every $\alpha$ is compatible with $\Pi$, and the proof of Theorem 1 simplifies because in the above construction $y_{E^{i}\left(\alpha\left(s^{i}\right)\right)}$ reduces to $y$.

\section{Incentive Compatibility}

It is clear that besides Bayesian monotonicity, implementability will also require some type of incentive compatibility condition, since (i) of Definition 4 requires every $x \in F$ to be an equilibrium outcome to the mechanism. The literature on Bayesian incentive compatibility (e.g. Myerson (1979) or Harris and Townsend (1981)) yields the result that $x$ is an equilibrium outcome to some mechanism if and only if $x$ satisfies certain incentive compatibility conditions. The version of incentive compatibility required for our purposes is the following.

Definition 13: $F$ satisfies Incentive Compatibility (IC) if, for all $x \in F$, for all $i$, for all $s$, for all $E^{i} \in \Pi^{i}$,

$$
x R^{i}\left(E^{i}(s)\right) x_{E^{\prime}}
$$

It is straightforward to show that if $x$ is an equilibrium outcome to some mechanism, then $x$ satisfies IC. Formally, we have the following theorem. 
THEOREM 2: If $F$ is implementable, $F$ satisfies IC.

Proof: Let $x \in F$. Part (i) of Definition 4 requires that $x$ be an equilibrium outcome. By the Revelation Principle, there is a direct mechanism to which truth is an equilibrium and $x$ is the truthful equilibrium outcome. Let $h$ denote the allocation rule in this direct mechanism. Consider the alternative direct mechanism $g$ with

$$
\begin{aligned}
& g\left(E^{1}, \ldots, E^{I}\right)=h\left(E^{i}, \ldots, E^{I}\right) \text { if } \bigcap_{i} E^{i} \neq \varnothing, \\
& g\left(E^{1}, \ldots, E^{I}\right)=0 \quad \text { otherwise. }
\end{aligned}
$$

Then, truth continues to be an equilibrium to $g$, and $x$ remains the truthful equilibrium outcome. Since for any $i$ and $E^{i} \in \Pi^{i}, x_{E^{i}}$ is the outcome of the game when all agents except $i$ tell the truth, and $i$ reports $E^{i}$, we must have $x R^{i}\left(E^{i}(s)\right) x_{E^{i}}$ for all $i$, which is precisely the inequality in IC.

Q.E.D.

Again, as in Theorem 1, the proof for diffuse information structures is very simple since the definition of incentive compatibility reduces to the "self-selection" condition of Harris and Townsend (1981) and the Bayesian incentive compatibility condition of Myerson (1979).

\section{SUFFICIENCY}

The previous Section identified two intuitive conditions which are necessary for a set of allocation rules to be fully implementable in Bayesian equilibrium. In this Section, we show that when there are at least three agents, essentially the same conditions are sufficient for implementation. For reasons made clear below, our proof of sufficiency uses the following modified incentive compatibility condition.

For any $\varepsilon \in A$ such that $\varepsilon \neq 0$, let

$$
x_{E^{i}}^{\varepsilon}(t)= \begin{cases}x\left(E^{-i}(t) \cap E^{i}\right) & \text { if } E^{-i}(t) \cap E^{i} \neq \varnothing, \\ \varepsilon & \text { otherwise. }\end{cases}
$$

Definition 14: $F$ satisfies $\varepsilon$-Incentive Compatibility $(\varepsilon-I C)$ if for all $x \in F$, for all $i$, for all $s$, for all $E^{i} \in \Pi^{i}$,

$$
x R^{i}\left(E^{i}(s)\right) x_{E^{i}}^{\varepsilon}
$$

This condition, which is a slight strengthening of IC, is equivalent to IC in a large class of models. For example, this constraint reduces to IC whenever information is diffuse. 
Theorem 3: If $I \geq 3$, and $F$ is Bayesian monotonic and $\varepsilon-I C$ for some $\varepsilon>0$, then $F$ is implementable.

Proof: We construct a mechanism to implement $F$. Note first that if $F$ satisfies $\varepsilon-I C$, then $F$ satisfies $\varepsilon^{\prime}-I C$ for all $\varepsilon^{\prime}<\varepsilon$. Thus without loss of generality, we can assume that $F$ satisfies $\varepsilon-I C$ where $\varepsilon<\bar{w} / I$. Let

$$
M^{i}=\Pi^{i} \times X \times\{0,1,2,3, \ldots\},
$$

and

$$
M=M^{i} \times M^{2} \times \ldots \times M^{I} .
$$

We define $g$ separately for different subsets of $M$. Let

$$
\begin{aligned}
& D_{1}=\left\{m \mid m^{i}=\left(E^{i}, x, n^{i}\right) \forall i ; x \in F ; \varnothing \neq \bigcap_{i} E^{i}=\{\bar{s}\} ;\right. \\
& \left.\quad n^{i} \neq 1 \forall i ; n^{i}=0 \text { for at least } I-1 \text { agents }\right\}, \\
& D_{2}(i)=\left\{m \mid m^{j}=\left(E^{j}, x, 0\right) \forall j \neq i ; x \in F ; m^{i}=\left(E^{i}, y, 1\right) ; \bigcap_{j \neq i} E^{j} \neq \varnothing\right\}, \\
& D_{2}=\bigcup_{i} D_{2}(i), \\
& D_{3}(i)=\left\{m \mid m^{j}=\left(E^{j}, x, 0\right) \forall j \neq i ; x \in F ; \bigcap_{j \neq i} E^{j} \neq \varnothing ; m \notin D_{1} \cup D_{2}\right\}, \\
& D_{3}=\bigcup_{i} D_{3}(i), \\
& D_{4}=\left\{m \mid m \notin D_{1} \cup D_{2} \cup D_{3}\right\} .
\end{aligned}
$$

We further subdivide $D_{2}(i)$ as follows:

$$
\begin{aligned}
D_{a}(i)= & \left\{m \in D_{2}(i) \mid \varnothing \neq \bigcap_{j} E^{j}=\{\bar{s}\} ; x R^{i}\left(E^{i}(s)\right) y_{E^{i}} \forall s \in \bigcap_{j \neq i} E^{j}\right\}, \\
D_{b}(i)= & \left\{m \in D_{2}(i) \mid \varnothing \neq \bigcap_{j} E^{j}=\{\bar{s}\} ;\right. \\
& \left.y_{E^{i}} P^{i}\left(E^{i}(s)\right) x \text { for some } s \in \bigcap_{j \neq i} E^{j}\right\}, \\
D_{c}(i)= & \left\{m \in D_{2}(i) \mid \bigcap_{j} E^{j}=\varnothing\right\} .
\end{aligned}
$$

Note that if $D_{a}(i) \cup D_{b}(i) \cup D_{c}(i)=D_{2}(i)$, and for $j \neq i, D_{2}(i) \cap D_{2}(j)=\varnothing$. We subdivide $D_{3}$ as follows:

$$
\begin{aligned}
& D_{31}=\left\{m \in D_{3} \mid m_{3}^{i}=0 \text { for all } i \text { or there exist } j, k \text { with } m_{2}^{j} \neq m_{2}^{k}\right\}, \\
& D_{32}=\left\{m \in D_{3} \mid m_{3}^{i}>1 \text { for some } i \text { and } m_{2}^{j}=m_{2}^{k} \text { for all } j, k\right\} .
\end{aligned}
$$


Note that if $m_{3}^{i}=1$, the aggregate message is in $D_{2}(i)$. Finally, let

$$
\bar{k}=\max \left\{m_{3}^{i} \mid i=1,2, \ldots, I\right\}, K=\left\{i \mid m_{3}^{i}=\bar{k}\right\}, k=\# K .
$$

We define $g: M \rightarrow A$ as follows:

$$
\begin{array}{ll}
g(m)=x(\bar{s}), & \text { if } m \in D_{1}, \\
g(m)=y(\bar{s}), & \text { if } m \in D_{a}(i) \text { for some } i, \\
g(m)=0, & \text { if } m \in D_{b}(i) \text { for some } i, \\
g(m)=0, & \text { if } m \in D_{c}(i) \text { for some } i, \\
g(m)=0, & \text { if } m \in D_{31}, \\
g^{i}(m)=\varepsilon, & \text { if } m \in D_{32} \text { and } m_{3}^{i}>1, \\
g^{j}(m)=0, & \text { if } m \in D_{32} \text { and } m_{3}^{j}=0, \\
g^{i}(m)= \begin{cases}\bar{w} / k, & \text { if } m \in D_{4} \text { and } i \in K, \\
0, & \text { if } m \in D_{4} \text { and } i \notin K .\end{cases}
\end{array}
$$

Note that $g$ is 'wasteful', in that it appropriates resources in $D_{3}$ and in $D_{2}$.

\section{Explanation of the Mechanism}

The message space is divided into four basic regions, $D_{1}-D_{4} . D_{1}$ is a region of agreement: the reports of agents agree on a state, $\bar{s}$, all agents request the same allocation rule, $x$ (in $F$ ), and at most one agent reports a positive integer. In this region, the outcome is $x(\bar{s})$. In $D_{2}(i)$, agent $i$ deviates unilaterally from $D_{1}$ by reporting a " 1 " and asking for some $y \in X$. In this region, the outcome depends on whether the message lies in $D_{a}(i), D_{b}(i)$, or $D_{c}(i)$. In $D_{a}(i), i$ appears no better off with $y$ than with $x$ at all states collectively reported by $j \neq i$ and in this case, the outcome is $y(\bar{s})$, where the reported events of all agents agree on $\bar{s}$. In $D_{b}(i), i$ is better off with $y$ than with $x$ at some state collectively reported by the other agents, while in $D_{c}(i)$, there is a disagreement about the state. In both $D_{b}(i)$ and $D_{c}(i)$, all resources are appropriated. $D_{3}$ is divided into two subregions. In $D_{31}$, either all agents submit zero or some agent requests $y \neq x$ but fails to submit a " 1 ". In $D_{32}$, exactly one agent submits a positive integer greater than one and all agents request the same allocation rule. From the definition of $D_{3}$, this is an exhaustive partition of $D_{3}$. In $D_{31}$, all resources are appropriated, while in $D_{32}$ the agent submitting a positive integer gets $\varepsilon$ while all other resources are appropriated. This outcome function prevents any equilibrium in $D_{31}$ : by reporting an integer greater than one, some agent can move from $D_{31}$ to either $D_{32}$ or $D_{4}$ and be better off. Finally, $D_{4}$ is a region of multilateral deviations from $D_{1}$; here, the agent reporting the highest integer receives all the resources. Since any $j \neq i$ can move unilaterally from $D_{2}(i)$ and $D_{32}$ to $D_{4}$, there is no equilibrium in any of these regions. There is clearly no equilibrium in $D_{4}$. Thus, all equilibria lie in $D_{1}$. 
The above intuition is formalized in three lemmas which together constitute a proof of Theorem 3. Proofs are in the Appendix. The first lemma establishes that no equilibrium message lies outside of $D_{1}$ and that no positive integer is used.

Lemma 2: If $\sigma$ is an equilibrium to $(M, g)$, then $\sigma(E(s)) \in D_{1}$ for all $s$ and $\sigma_{3}^{i}\left(E^{i}(s)\right)=0$ for all $i$ and $s$.

The next lemma shows that any strategy profile in which every agent truthfully reports his event, requests the same element of $F$, and submits the integer 0 , is an equilibrium. This establishes that the mechanism (weakly) implements $F$. That is, condition (i) of the definition of implementation is satisfied:

For any $x \in F$, there exists an equilibrium to $(M, g)$ such that $g(\sigma)=x$.

Lemma 3: For any $x \in F, \sigma^{i}\left(E^{i}(s)\right)=\left(E^{i}(s), x, 0\right)$ for all $i$ and for all $s$ is an equilibrium.

Finally, the last lemrina establishes that $(M, g)$ fully implements $F$.

Lemma 4: If $\sigma$ is an equilibrium to $(m, g)$, then $g(\sigma) \in F$.

A few remarks about the proof of Theorem 3 are in order. First, the mechanism we construct to implement an SCS is related to the standard Maskin-type mechanism (Maskin (1977), Williams (1984), Saijo (1988), McKelvey (1985)), but differs in some important ways. One similarity is that all players are asked to reveal what they know about others as well as what they know about themselves (since reporting an event is like reporting a subset of possible preference profiles). in fact the rest of the message space, an allocation rule and an integer, also parallels the mechanisms used to implement SCS's with complete information. It differs from the complete information mechanisms in two ways. First everyone is severely punished in $D_{3}$. The reason for this is that for some disagreement messages the planner may be unable to detect who is "causing" the disagreement. That is, a unilateral departure from a compatible joint strategy can be made anonymously. This is illustrated by a four-state example. Let $\Pi^{1}=$ $\{(1,2),(3,4)\}, \Pi^{2}=\{(1,3),(2,4)\}, \Pi^{3}=\{(1,4),(2,3)\}$. Suppose $\alpha^{1}$ and $\alpha^{2}$ are identity maps, and $\alpha^{3}\{(2,3)\}=(1,4), \alpha^{3}\{(1,4)\}=(2,3)$. When state 1 occurs, 1 and 2 report $(1,2)$ and $(1,3)$ respectively, while 3 reports $(2,3)$. Then, the intersection of the reports of any $I-1$ group is nonempty, while the overall intersection is empty. In this case, it is impossible to detect the deviator. In fact, most of the apparent complexity of our mechanism can be attributed to this type of problem, arising because of possible incompatibilities in deceptive strategies. Most of these complications disappear if the information structure falls in one of two classes: (i) complete information, when all unilateral deceptions are incompatible, and (ii) diffuse information structures, in which all deceptions are compatible. In the (more interesting) second case, the mechanism and proof become much simpler since in this case the region $D_{3}$ can be combined with $D_{1}$ 
while $D_{c}(i)$ is empty for all $i$. The reason for this is that regions $D_{3}$ and $D_{c}(i)$ only come into play when there are incompatible reports, but if information is diffuse, all reports are compatible. The elimination of these two regions removes the need to use collective punishments, and so permits nonwasteful implementation.

Second, each player requests a state-contingent allocation rather than a single noncontingent allocation, as in the complete information case. The reason this is needed is very simple. Since players make their decisions at an interim stage, they are concerned with possible allocations in more than one state (i.e., all states belonging to the event they observe). Therefore, the agents will have to be required to request allocations which are contingent on at least all those states in the relevant common knowledge event. Of course, this reduces to a single state under complete information.

The $\varepsilon$-qualification of incentive compatibility, weak as it is, is stronger than what is needed in our constructive proof. The role of this qualification is to rule out equilibria in $D_{31}$. In our mechanism, an arbitrarily small incentive $(\varepsilon)$ is available to ensure that some agent, say $j$, has an incentive to move the aggregate message from $D_{31}$ to $D_{32}$. In turn, $\varepsilon-I C$ is then required in the proof of Lemma 3 to remove an incentive for that agent to move the aggregate message from $D_{1}$ to $D_{32}$. All that is needed for this argument to work is that in each state, $\varepsilon-I C$ hold for at least one player, and $I C$ hold for everyone else. However, as we pointed out above, $\varepsilon-I C$ reduces to $I C$ in most cases of interest.

Finally, we comment on the role of three or more agents. The reason we assume $I \geq 3$ is the same as the reason it is usually assumed in the case of complete information and has to do with the particular construction used in the proof. It is used in Lemma 2 to ensure that if the aggregate message lies in $D_{3}(i)$ for all $i$, then there is at least one agent who can profitably deviate by reporting a large integer. In our construction, this is not always possible if $I=2$.

\section{IDENTIFYING IMPLEMENTABLE ALLOCATIONS: SOME EXAMPLES}

In this Section, we provide examples of SCS's which are and are not implementable and relate our findings to the literature on implementation with complete information. In studies of implementation via Nash equilibrium in complete information economies, a central result relates implementable SCS's to Walrasian equilibrium allocations. It is well known (Hurwicz (1979), Schmeidler (1980), Hurwicz, Maskin, and Postlewaite (1984)) that the SCS which associates the set of (constrained) Walrasian allocations to each state is implementable. In economies with incomplete information, one might hope that a similar result holds. However, a problem arises concerning what sort of "similar" result to look for. Specifically, one must ask what is the appropriate extension of Walrasian allocations to economies with incomplete information. Two possibilities immediately come to mind:(1) Arrow-Debreu complete contingent claims allocations, and (2) rational expectations equilibria in which a trader's informational constraints are assumed to be relaxed by public price information. 
In considering the first of these, it is worth noting that the Arrow-Debreu allocations are ex-ante Pareto optimal since insurance is made possible by trading across states. However, the implicit timing of the resolution of uncertainty in the Arrow-Debreu model is different from the timing in our framework. In our model, trades are made after agents receive their private information. Consequently, in our model, Arrow-Debreu allocations may well fail to be individually rational.

Rational expectations equilibrium allocation rules correspond much more closely to the timing structure we use. The main difference between our model and rational expectations equilibrium models is that in the course of playing out a mechanism, we do not assume that an agent's information is augmented by any aggregate statistic of other traders' messages (such as a price). Rather, each agent plays the game knowing only which event he has observed.

EXAmple 1: (Arrow-Debreu allocation rules are not always implementable): There are two informed agents, one good and two states. The parameters are: $w^{1}=(3,3), w^{2}=(2,2), U^{1}\left(x^{1}, s\right)=s \cdot \log \left(x^{1}\right), s=1,2, U^{2}\left(x^{2}, s\right)=\log \left(x^{2}\right), q^{1}=$ $q^{2}=(.5, .5)$. Then, the unique equilibrium state prices from trading in a complete contingent claims market are $(1,3 / 2)$ and the equilibrium allocation rule is given by:

\section{State $1 \quad$ State 2}

$\begin{array}{llr}\text { Agent } 1 & 5 / 2 & 10 / 3 \\ \text { Agent } 2 & 5 / 2 & 5 / 3\end{array}$

Let $F$ be the SCS whose values are given in the above table. We will show that $F$ does not satisfy Bayesian monotonicity. Consider $\alpha=\left(\alpha^{1}, \alpha^{2}\right)$ with $\alpha^{i}(t)=\{1\}$ for both $i$ and for both $t$. Then, $\alpha$ is compatible with $\Pi$ since both agents are perfectly informed, and $\alpha(t)=\{1\}$ for all $t$. Since both agents are perfectly informed and utility is increasing, the Bayesian monotonicity hypothesis is trivially satisfied. For example, for $s^{\prime}=2$, the condition for agent 1 reads $U^{1}\left(x^{1}(1), 1\right) \geq U^{1}\left(y^{1}(1), 1\right)$ implies $U^{1}\left(x^{1}(1), 2\right) \geq U^{1}\left(y^{1}(1), 2\right)$. Bayesian monotonicity then requires that $(x(1), x(1))$ must lie in $F$. However, $(x(1), x(1))$ is not an Arrow-Debreu equilibrium. Since Bayesian monotonicity is necessary for implementation, this implies that the Arrow-Debreu complete contingent claims equilibrium is not implementable.

The reason why $F$ fails to satisfy Bayesian monotonicity is intuitively clear. The Arrow-Debreu allocation rules are ex-ante allocation rules, and thus provide insurance, while the structure of the game analyzed in the previous Sections requires trades to be made after agents receive their private information. Once all agents are perfectly informed, there is no practical possibility for insurance. Not surprisingly, then, this allocation rule can fail to be implementable. 
While the intuition behind this example is straightforward, it is interesting to examine the consequences of adding an uninformed agent to the above economy. In the next example, we add such an agent, and specify his preferences and endowments so that this agent does not change the equilibrium allocations of agents 1 and 2. Somewhat surprisingly, the allocation rule now becomes implementable.

EXAMPLE 2 (Adding an uninformed agent makes the allocation rule implementable): Consider the above example with the addition of a completely uninformed agent, say agent 3 , with $\Pi^{3}=\{1,2\}, w^{3}=(1,1)$, and $U^{3}\left(x^{3}, s\right)=$ $(s+1) \log \left(x^{3}\right)$. With these parameters, the Arrow-Debreu SCS is given by:

\section{State $1 \quad$ State 2}

$\begin{array}{ccc}\text { Agent } 1 & 5 / 2 & 10 / 3 \\ \text { Agent 2 } & 5 / 2 & 5 / 3 \\ \text { Agent 3 } & 1 & 1\end{array}$

Here, the uninformed agent receives his initial endowment at the equilibrium. Thus, agent 3 adds "nothing" to the economy in any real or informational sense. Nonetheless, we now show that with the addition of the uninformed agent, the SCS satisfies Bayesian monotonicity. Consider $\alpha^{1}$ and $\alpha^{2}$ as in Example 1. $\alpha^{3}$ is constrained to be the identity since agent 3 is uninformed. As above, the Bayesian monotonicity hypothesis is trivially satisfied for agents 1 and 2 , so consider the hypothesis for agent 3 with $E^{3}=\{1,2\}$ :

$$
\begin{aligned}
& q^{3}(1) U^{3}\left(x^{3}(1), 1\right)+q^{3}(2) U^{3}\left(x^{3}(2), 2\right) \\
& \geq q^{3}(1) U^{3}\left(y^{3}(1), 1\right)+q^{3}(2) U^{3}\left(y^{3}(2), 2\right)
\end{aligned}
$$

implies

$$
\begin{aligned}
& q^{3}(1) U^{3}\left(x^{3}(1), 1\right)+q^{3}(2) U^{3}\left(x^{3}(1), 2\right) \\
& \geq q^{3}(1) U^{3}\left(y^{3}(1), 1\right)+q^{3}(2) U^{3}\left(y^{3}(1), 2\right) .
\end{aligned}
$$

If we pick $y^{3}(1)=2, y^{3}(2)=.02$, then (a) is satisfied while (b) is not. For this $\alpha$, then, Bayesian monotonicity does not restrict $F$. A similar argument applies to the $\alpha$ in which both informed agents always agree to 2 . For the $\alpha$ in which $\alpha^{i}\{(1)\}=(2), \alpha^{i}\{(2)\}=(1), i=1,2$, let $y^{3}(1)=1.5, y^{3}(2)=.75$, and again, the first inequality is satisfied but the second is not. Therefore Bayesian monotonicity is satisfied. Further, it is straightforward to check that there exists an $\varepsilon$ such that $\varepsilon-I C$ is satisfied. For example, for agent 1 at $s=1$, it reduces to $U^{1}(x(1), 1) \geq$ $U^{1}(x(1), 1)$ if $E^{1}=\{1\}$ and $U^{1}(x(1), 1) \geq U^{1}(\varepsilon, 1)$ if $E^{1}=\{2\}$. Similar inequalities follow at $s=2$ and for agent 2. Since $x$ is strictly positive, there exists an $\varepsilon$ so that $\varepsilon-I C$ is satisfied for both agents 1 and 2 . For agent $3, E^{3}(s)=\{1,2\}$ for all $s$, so the condition reduces to $x R^{i}\left(E^{i}(s)\right) x$ for all $s$. Since $I=3$, this allocation rule is implementable. In fact, using the mechanism in the proof of Theorem 3 , it is easily seen that the strategy described in Lemma 3 is the only equilibrium. 
The intuition behind this example is quite straightforward. Consider the $\alpha$ which led to a violation of Bayesian monotonicity in Example 1, in which both informed agents always report state 1 . In our mechanism, agent 3 can simply report $y=(2,0.02)$ and get 2 in either state. Thus, $(x(1), x(1))$ is no longer an equilibrium outcome in this example while it was one in Example 1. From this it is also clear that any direct mechanism to which $x$ is an equilibrium outcome in Example 1 will also have $(x(1), x(1))$ as an equilibrium outcome, since in this case, agent 3 cannot affect the outcome.

The rational expectations equilibrium SCS (see, for example, Radner (1979)) in the above examples consists of the initial endowments since there is only one good, and is (trivially) implementable. More generally, if individual endowments are state independent and all rational expectations equilibrium allocations are strictly interior, then the rational expectations equilibrium SCS is implementable when there are at least three agents and every group of $I-1$ agents collectively has complete information. This is shown in Palfrey and Srivastava (1987), where it is also shown that neither state independence of endowments nor interiority can generally be relaxed. Furthermore, rational expectations equilibrium allocation rules generally are not incentive compatible (Blume and Easley (1985)), and therefore they will not satisfy $\varepsilon-I C$.

\section{CONCLUSIONS}

This paper characterized implementable SCS's in pure exchange economic environments with no aggregate endowment uncertainty when individuals have asymmetric incomplete information about the state. An incentive compatibility condition is required for well-known reasons relating to the revelation principle. A monotonocity condition is required for the same reasons it is required with complete information. This demonstrates that the characterization of (Nash) fully implementable allocations with complete information extends in a very natural way to incomplete information.

Despite this apparent similarity between complete and incomplete information, we show via some examples in Section 5 that some of the "intuitive" interpretations of implementable allocations with complete information do not extend in an obvious way to incomplete information. In particular, we provide some examples in which SCS's with incomplete information are not implementable.

One extension not addressed in the paper is the implementation problem in more general environments than the pure exchange setting examined here. While we present no results here, a few comments can be made. First, for sufficiency we will certainly need a no veto power condition, appropriately modified to account for incomplete information. Such a condition requires that an outcome be included in the SCS if it is nearly unanimously (except for possibly 1 person) viewed as the best outcome. Second, our construction depends upon our ability to identify an outcome which is unanimously bad for all individuals in all states (the 0 allocation). It appears that in the general case some condition relating to a worst outcome may be required unless information is diffuse. On the other hand, 
extending the necessity results to more general environments is simpler. If information is diffuse, the necessity arguments of both Theorem 1 and Theorem 2 apply directly, since incompatible reports do not occur so reliance on "worst" outcomes and collective punishments is removed. If information is not diffuse, then the monotonicity condition (and IC) will have to be modified because a collective punishment may not be available. A complete characterization of fully implementable allocation rules under incomplete information in general (noneconomic) environments remains an open question.

Finally, we remark on the specification of a state. In our model, in Postlewaite and Schmeidler (1986), and implicitly in the literature on implementation with complete information, the information structure is not a part of the description of a state, but is known to the planner. This is assumed implicitly in our proof of sufficiency since a part of the message space of an agent is his partition. It would appear fruitful to extend the analysis to the case where the partitions are also private information but in a sense such an extension would only be definitional. It can be done by treating the information structure as a part of the specification of a state, in which case an SCS would map the set of possible partitions and preferences into allocations. We also assumed throughout the analysis that there was no private information about endowments (see Postlewaite (1979)), and relaxing this assumption would also appear to be a fruitful direction to pursue.

Division of Humanities and Social Sciences, California Institute of Technology, Pasadena, CA 91125, U.S.A.

and

Graduate School of Industrial Administration, Carnegie-Mellon University, Pittsburgh, PA 15213-3890, U.S.A.

Manuscript received March, 1985; final revision received February, 1988.

\section{APPENDIX}

Proof of Lemma 2: We first show that $\sigma\left((E(s)) \in D_{1}\right.$, for all $s$. Suppose not. Then, there are four cases to consider: (1) $\sigma(E(s)) \in D_{2}(i)$ for some $i, s ;(2) \sigma(E(s)) \in D_{32}$ for some $s ;(3) \sigma(E(s)) \in D_{31}$ for some $s ;(4) \sigma(E(s)) \in D_{4}$ for some $s$.

CASE 1: In this case, there exists an $i$ and an $s$ such that $\sigma(E(s)) \in D_{2}(i)$. Since $I \geq 3$, there exists $j \neq i$ who observes $E^{j}(s)$ and who does not receive $\bar{w}$ under $g$ at $s$. For this $j$, define

$$
\begin{aligned}
& \tilde{\sigma}_{1}^{j}\left(E^{j}(t)\right)=\sigma_{1}^{j}\left(E^{j}(t)\right), \text { all } t, \\
& \tilde{\sigma}_{2}^{j}\left(E^{j}(t)\right)=\sigma_{2}^{j}\left(E^{j}(t)\right), \text { all } t, \\
& \tilde{\sigma}_{3}^{j}\left(E^{j}(s)\right)=2+\max \left\{\sigma_{3}^{k}\left(E^{k}(t)\right) \mid k=1,2, \ldots, I ; t \in S\right\}, \\
& \tilde{\sigma}_{3}^{j}\left(E^{j}(t)\right)=\sigma_{3}^{j}\left(E^{j}(t)\right) \text { for all } t \notin E^{j}(s) .
\end{aligned}
$$

Under $\tilde{\sigma}^{j}, j$ reports the same events and requests the same allocation rule as before, but at $E^{j}(s)$, he reports a number which is not equal to one and is guaranteed to be larger than that reported by any other agent. 
Claim: By using $\tilde{\sigma}^{j}$, agent $j$ is strictly better off when he observes $E^{j}(s)$. To see this, define $\tilde{\sigma}=\left(\sigma^{-j}, \tilde{\sigma}^{j}\right)$, and consider any $t \in E^{j}(s)$. There are five subcases to consider:

(i) If $\sigma(E(t)) \in D_{1}$, then either $\tilde{\sigma}(E(t)) \in D_{1}$, in which case $j$ is just as well off as under $\sigma^{j}$, or $\tilde{\sigma}(E(t)) \in D_{4}$, in which case $j$ receives $\bar{w}$, and is at least as well off.

(ii) If $\sigma(E(t)) \in D_{2}(k)$ for some $k$, then $\tilde{\sigma}(E(t)) \in D_{4}, j$ gets $\bar{w}$ and is at least as well off. Note that since $\sigma(E(s)) \in D_{2}(i)$, it cannot be the case that $\sigma(E(t)) \in D_{2}(j)$. At $s$, when $k=i, j$ is strictly better off.

(iii) If $\sigma(E(t)) \in D_{31}$, then $g^{j}(\sigma(E(t))=0$ so $j$ cannot possibly be worse off.

(iv) If $\sigma(E(t)) \in D_{32}$ and $j$ is not reporting a positive integer under $\sigma$, then $\tilde{\sigma}(E(t)) \in D_{4}, j$ gets $\bar{w}$ and is strictly better off. If $\sigma(E(t)) \in D_{32}$ and $j$ is reporting a positive integer, then $\tilde{\sigma}(E(t)) \in D_{32}$ and there is no change.

(v) If $\sigma(E(t)) \in D_{4}$, then $\tilde{\sigma}(E(t)) \in D_{4}, j$ gets $\bar{w}$, and is at least as well off.

In each subcase, therefore, $j$ is either strictly better off or at least as well off, and at $s$ he is strictly better off. This completes the proof of the claim that if, for some $s, \sigma(E(s)) \in D_{2}(i)$, then $\sigma$ cannot be an equilibrium.

In case 2 , there exists an $s$ such that $\sigma(E(s)) \in D_{3}(i)$ and a $j$ such that $\sigma(E(s)) \notin D_{3}(j)$. In this case, we can define $\tilde{\sigma}^{j}$ exactly as above and can show that $j$ is strictly better off using $\tilde{\sigma}^{j}$.

In case 3 , we define $\tilde{\sigma}^{j}$ in the same way as in case 1 using some $j$ for whom $\sigma(E(s)) \in D_{3}(j)$. Then, at $s, j$ moves the aggregate message from $D_{31}$ to $D_{32}$ and gets $\varepsilon$ instead of 0 , in which case $j$ is strictly better off. It is again straightforward to show that $j$ is at least as well off at all $t \in E^{J}(s)$. In case 4 , defining $\tilde{\sigma}^{j}$ in the same way for some $j$ who does not get $\bar{w}$ at $s$ yields that $j$ is strictly better off using $\tilde{\sigma}^{j}$, so that $\sigma$ could not have been an equilibrium.

We next show that no positive integers are used. From above, $\sigma(E(s)) \in D_{1}$ for all $s$. If $\sigma_{3}(E(s)) \neq 0$ for $s$, then define $\tilde{\sigma}^{j}\left(E^{j}(s)\right)$ as above for some $j$ who does not get $\bar{w}$ at $s$. Then, following the same argument as before, it can be shown that $j$ is strictly better off using $\tilde{\sigma}^{j}$ instead of $\sigma^{j}$, so $\sigma$ cannot be an equilibrium.

Proof of Lemma 3: Suppose $I-1$ agents are using the above strategy. Consider agent $i$. If, at $s, i$ submits the message $\left(E^{i}(s), x, 0\right)$, then the outcome at $t \in E^{i}(s)$ is $x(t)$. Suppose instead that $i$ uses $\left(E^{i}, y, n\right) \neq\left(E^{i}(s), x, 0\right)$ at $s$. There are several cases to consider:

(i) $n \neq 1, y=x$ : In this case, $m \in D_{1}$ if $E^{-i}(t) \cap E^{i} \neq \varnothing$ and $m$ lies in $D_{32}$ or $D_{31}$ if $E^{-i}(t) \cap E^{i}$ $=\varnothing$, so at best, $i$ receives $x_{E^{\prime}}^{\varepsilon}$. From $\varepsilon-I C, x R^{i}\left(E^{i}(s)\right) x_{E^{\prime}}^{\varepsilon}$, so $\left(E^{i}(s), x, 0\right)$ does at least as well as $\left(E^{i}, y, n\right)$.

(ii) $n \neq 1, y \neq x$ : In this case, $m \in D_{31}$ for all $t \in E^{i}(s), i$ gets 0 at all $t \in E^{i}(s)$, so that submitting $\left(E^{i}(s), x, 0\right)$ does at least as well for $i$.

(iii) $n=1$ : In this case, $m \in D_{2}(i)$ for all $t \in E^{i}(s)$. Let

$$
\begin{aligned}
& T_{a}=\left\{t \in E^{i}(s) \mid m \in D_{a}(i)\right\}, \\
& T_{b}=\left\{t \in E^{i}(s) \mid m \in D_{b}(i) \cup D_{c}(i)\right\} .
\end{aligned}
$$

Let $z(t)$ be the outcome at $t$. Then, $z(t)=y_{E^{\prime}}(t)$ if $t \in T_{a}$ and $z(t)=0$ if $t \in T_{b}$. Since preferences are increasing, $y_{E^{\prime}} R^{i}\left(E^{i}(s)\right) z$. Since all $j \neq i$ are reporting $E^{j}(s), s \in \cap_{j \neq l} E^{j}$, and $m \in D_{a}(i)$ requires $x R^{i}\left(E^{i}(s)\right) y_{E^{\prime}}$. Thus, $x R^{i}\left(E^{i}(s)\right) z$, and $\left(E^{i}(s), x, 0\right)$ does at least as well as $\left(E^{i}, y, n\right)$.

Q.E.D.

Proof of Lemma 4: Let $\sigma$ be any equilibrium to $(M, g)$. Then, by Lemma $2, \sigma(E(s)) \in D_{1}$ for all $s$. Since $F$ is closed under the common knowledge concatenation defined by $\Pi$ (Assumption 2), we get $\sigma_{2}^{i}\left(E^{i}(s)\right)=x \in F$ for all $i$ and $s$. Define $\alpha^{i}\left(E^{i}(s)\right)=\sigma_{1}^{i}\left(E^{i}(s)\right), i=1,2, \ldots, I$. Then, $\alpha$ is compatible with $\Pi$, and the equilibrium outcome is $x_{\alpha}$. We have to show that $x_{\alpha} \in F$.

Suppose not. Then Bayesian monotonicity yields $i, s^{\prime}, y$ such that $x R^{i}\left(E^{\prime}(s)\right) y_{E^{\prime}\left(\alpha\left(s^{\prime}\right)\right)}$ for all $s \in E_{\alpha}^{-i}\left(s^{\prime}\right)$ but $y_{\alpha} P^{i}\left(E^{i}\left(s^{\prime}\right)\right) x_{\alpha}$. Then, using $\sigma^{i}$ when $i$ observes $E^{\prime}\left(s^{\prime}\right)$ is not a best response for $i$; if, at $E^{i}\left(s^{\prime}\right)$, he reports $\left(\alpha^{i}\left(E^{i}\left(s^{\prime}\right)\right), y, 1\right)$, he moves from $D_{1}$ to $D_{a}(i)$, the outcome is $y_{\alpha}$ instead of $x_{\alpha}$, and $i$ is strictly better off. This is true no matter which state in $E^{i}\left(s^{\prime}\right)$ has occurred since by Lemma $1, \alpha(\tau) \in E^{i}\left(\alpha\left(s^{\prime}\right)\right)$ for all $\tau \in E^{i}\left(s^{\prime}\right)$. This contradicts $\sigma$ being an equilibrium, and so we get $x_{\alpha} \in F$. This completes the proof.

Q.E.D. 


\section{REFERENCES}

Blume, L., AND D. EASLeY (1985): “Implementation of Expectations Equilibria," mimeo, Cornell University.

Cramton, P., R. Gibbons, and P. Klemperer (1987): “Dissolving a Partnership Efficiently," Econometrica, 55, 615-632.

Dasgupta, P., P. HAMmond, AND E. MASKIn (1979): “The Implementation of Social Choice Rules: Some General Results on Incentive Compatibility," Review of Economic Studies, 46, 185-216.

DEMSKI, J., AND D. SAPPINGTON (1984): “Optimal Incentive Contracts with Multiple Agents," Journal of Economic Theory, 33, 152-171.

HARRIS, M., AND R. TOWNSEND (1981): "Resource Allocation Under Asymmetric Information," Econometrica, 49, 33-64.

HuRwicz, L. (1979): "Outcome Functions Yielding Walrasian and Lindahl Allocations at Nash Equilibrium Points," Review of Economic Studies, 46, 217-225.

HuRwicz, L., E. MaSkin, AND A. PostlewaIte (1984): "Feasible Implementation of Social Choice Correspondences by Nash Equilibria," mimeo.

KALAI, E., AND D. SAMET (1986): “Are Bayesian Nash Incentives and Implementations Perfect," mimeo, Northwestern University.

LAFFONT, J.-J., AND E. MASKIN (1982): “The Theory of Incentives: An Overview," in Advances in Economic Theory: Invited Papers for the Fourth World Congress of the Econometric Society, 1980, ed. by W. Hildenbrand. Cambridge: Cambridge University Press, pp. 31-94.

MASKIN, E. (1977): "Nash Equilibrium and Welfare Optimality," mimeo.

MCKELVEY, R. (1985): "Game Forms for Nash Implementation of General Social Choice Correspondences," Social Science Working Paper \#579, Caltech, forthcoming in Social Choice and Welfare.

Mirgrom, P. (1981): "Rational Expectations, Information Acquisition and Competitive Bidding," Econometrica, 49, 921-943.

Myerson, R., AND M. SATTERTHWAITE (1983): "Efficient Mechanisms for Bilateral Trading," Journal of Economic Theory, 29, 265-281.

- (1981): "Optimal Auction Design," Mathematics of Operations Research, 6, 58-73.

(1979): "Incentive Compatibility and the Bargaining Problems," Econometrica, 47, 61-73.

Palfrey, T., aNd S. SRIVASTAVA (1987): “On Bayesian Implementable Allocations," Review of Economic Studies, 54, 193-208.

Postlewaite, A. (1979): “Manipulation via Endowments," Review of Economic Studies, 46, 255-262.

Postlewaite, A., AND D. SChMEIDlER (1986): "Implementation in Differential Information Economies," Journal of Economic Theory, 39, 14-33.

RADNER, R. (1979): "Rational Expectations Equilibrium: Generic Existence and the Information Revealed by Prices," Econometrica, 47, 655-678.

RoB, R. (1984): “Pollution Claims Settlement Under Private Information," mimeo, University of Pennsylvania.

SaIJo, T. (1988): “Strategy Space Reduction in Maskin's Theorem: Sufficient Conditions for Nash Implementation," Econometrica, 56, 693-700.

SCHMEIDLER, D. (1980): “Walrasian Analysis via Strategic Outcome Functions," Econometrica, 48, $1585-1593$.

Williams, S. (1984): “Sufficient Conditions for Nash Implementation,” IMA Manuscript, University of Minnesota. 